\title{
The Complete Proof of the Riemann Hypothesis
}

\author{
Frank Vega
}

the date of receipt and acceptance should be inserted later

\begin{abstract}
Robin criterion states that the Riemann Hypothesis is true if and only if the inequality $\sigma(n)<e^{\gamma} \times n \times \log \log n$ holds for all $n>5040$, where $\sigma(n)$ is the sum-ofdivisors function and $\gamma \approx 0.57721$ is the Euler-Mascheroni constant. We prove that the Robin inequality is true for all $n>5040$ which are not divisible by any prime number between 2 and 953. Using this result, we show there is a contradiction just assuming the possible smallest counterexample $n>5040$ of the Robin inequality. In this way, we prove that the Robin inequality is true for all $n>5040$ and thus, the Riemann Hypothesis is true.
\end{abstract}

Keywords Riemann hypothesis · Robin inequality · sum-of-divisors function · prime numbers

Mathematics Subject Classification (2010) MSC 11M26 · MSC 11A41 · MSC $11 \mathrm{~A} 25$

\section{Introduction}

In mathematics, the Riemann Hypothesis is a conjecture that the Riemann zeta function has its zeros only at the negative even integers and complex numbers with real part $\frac{1}{2}$ [7]. As usual $\sigma(n)$ is the sum-of-divisors function of $n$ [3]:

$$
\sum_{d \mid n} d
$$

where $d \mid n$ means the integer $d$ divides to $n$ and $d \nmid n$ means the integer $d$ does not divide to $n$. Define $f(n)$ to be $\frac{\sigma(n)}{n}$. Say Robins $(n)$ holds provided

$$
f(n)<e^{\gamma} \times \log \log n .
$$

F. Vega

CopSonic, 1471 Route de Saint-Nauphary 82000 Montauban, France

ORCiD: 0000-0001-8210-4126

E-mail: vega.frank@gmail.com 
The constant $\gamma \approx 0.57721$ is the Euler-Mascheroni constant, and log is the natural logarithm. The importance of this property is:

Theorem 1.1 Robins $(n)$ holds for all $n>5040$ if and only if the Riemann Hypothesis is true [7].

It is known that Robins $(n)$ holds for many classes of numbers $n$.

Theorem 1.2 Robins $(n)$ holds for all $n>5040$ that are not divisible by 2 [3].

On the one hand, we prove that Robins $(n)$ holds for all $n>5040$ that are not divisible by any prime number between 3 and 953. Let $q_{1}=2, q_{2}=3, \ldots, q_{m}$ denote the first $m$ consecutive primes, then an integer of the form $\prod_{i=1}^{m} q_{i}^{a_{i}}$ with $a_{1} \geq a_{2} \geq \cdots \geq a_{m} \geq 0$ is called an Hardy-Ramanujan integer [3]. A natural number $n$ is called superabundant precisely when, for all $m<n$

$$
f(m)<f(n) .
$$

Theorem 1.3 If $n$ is superabundant, then $n$ is an Hardy-Ramanujan integer [2].

Theorem 1.4 The smallest counterexample of the Robin inequality greater than 5040 must be a superabundant number [1].

On the other hand, we prove the nonexistence of such counterexample and therefore, the Riemann Hypothesis is true.

\section{A Central Lemma}

These are known results:

Lemma 2.1 [3]. For $n>1$ :

$$
f(n)<\prod_{q \mid n} \frac{q}{q-1}
$$

Lemma $2.2[4]$.

$$
\prod_{k=1}^{\infty} \frac{1}{1-\frac{1}{q_{k}^{2}}}=\zeta(2)=\frac{\pi^{2}}{6} .
$$

The following is a key lemma. It gives an upper bound on $f(n)$ that holds for all $n$. The bound is too weak to prove Robins $(n)$ directly, but is critical because it holds for all $n$. Further the bound only uses the primes that divide $n$ and not how many times they divide $n$.

Lemma 2.3 Let $n>1$ and let all its prime divisors be $q_{1}<\cdots<q_{m}$. Then,

$$
f(n)<\frac{\pi^{2}}{6} \times \prod_{i=1}^{m} \frac{q_{i}+1}{q_{i}} .
$$


Proof We use that lemma 2.1:

$$
f(n)<\prod_{i=1}^{m} \frac{q_{i}}{q_{i}-1} .
$$

Now for $q>1$,

$$
\frac{1}{1-\frac{1}{q^{2}}}=\frac{q^{2}}{q^{2}-1}
$$

So

$$
\begin{aligned}
\frac{1}{1-\frac{1}{q^{2}}} \times \frac{q+1}{q} & =\frac{q^{2}}{q^{2}-1} \times \frac{q+1}{q} \\
& =\frac{q}{q-1} .
\end{aligned}
$$

Then by lemma 2.2,

$$
\prod_{i=1}^{m} \frac{1}{1-\frac{1}{q_{i}^{2}}}<\zeta(2)=\frac{\pi^{2}}{6} .
$$

Putting this together yields the proof:

$$
\begin{aligned}
f(n) & <\prod_{i=1}^{m} \frac{q_{i}}{q_{i}-1} \\
& \leq \prod_{i=1}^{m} \frac{1}{1-\frac{1}{q_{i}^{2}}} \times \frac{q_{i}+1}{q_{i}} \\
& <\frac{\pi^{2}}{6} \times \prod_{i=1}^{m} \frac{q_{i}+1}{q_{i}}
\end{aligned}
$$

\section{About the $p$-adic order}

In basic number theory, for a given prime number $p$, the $p$-adic order of a natural number $n$ is the highest exponent $v_{p} \geq 1$ such that $p^{v_{p}}$ divides $n$. This is a known result:

Lemma 3.1 In general, we know that Robins $(n)$ holds for a natural number $n>5040$ that satisfies either $v_{2}(n) \leq 19, v_{3}(n) \leq 12$ or $v_{7}(n) \leq 6$, where $v_{p}(n)$ is the $p$-adic order of $n[5]$.

We know the following lemmas:

Lemma 3.2 [5]. Let $\prod_{i=1}^{m} q_{i}^{a_{i}}$ be the representation of $n$ as a product of primes $q_{1}<$ $\cdots<q_{m}$ with natural numbers as exponents $a_{1}, \ldots, a_{m}$. Then,

$$
f(n)=\left(\prod_{i=1}^{m} \frac{q_{i}}{q_{i}-1}\right) \times \prod_{i=1}^{m}\left(1-\frac{1}{q_{i}^{a_{i}+1}}\right) .
$$


Lemma 3.3 [5]. Let $n>e^{e^{23.762143}}$ and let all its prime divisors be $q_{1}<\cdots<q_{m}$, then

$$
\left(\prod_{i=1}^{m} \frac{q_{i}}{q_{i}-1}\right)<\frac{1771561}{1771560} \times e^{\gamma} \times \log \log n .
$$

Lemma 3.4 Robins $(n)$ holds for all $10^{10^{10}} \geq n>5040$ [5].

Putting together all these results, then we obtain that

Lemma 3.5 Robins $(n)$ holds for $n>5040$ when $v_{31}(n) \leq 3$.

Proof From lemma 3.2, we note that

$$
f(n)=\left(\prod_{i=1}^{m} \frac{q_{i}}{q_{i}-1}\right) \times \prod_{i=1}^{m}\left(1-\frac{1}{q_{i}^{a_{i}+1}}\right) \leq\left(\prod_{i=1}^{m} \frac{q_{i}}{q_{i}-1}\right) \times\left(1-\frac{1}{31^{v_{31}(n)+1}}\right)
$$

when $v_{31}(n) \leq 3$. We only need to look at the case where $v_{31}(n)=3$ since the weaker cases follow because

$$
\left(1-\frac{1}{31^{1+1}}\right)<\left(1-\frac{1}{31^{2+1}}\right)<\left(1-\frac{1}{31^{3+1}}\right)
$$

In this way, we obtain that

$$
f(n) \leq\left(\prod_{i=1}^{m} \frac{q_{i}}{q_{i}-1}\right) \times\left(1-\frac{1}{31^{3+1}}\right)=\frac{923520}{923521} \times\left(\prod_{i=1}^{m} \frac{q_{i}}{q_{i}-1}\right)
$$

when $v_{31}(n) \leq 3$. With lemma 3.3, we have for $n>e^{e^{23.762143}}$

$$
\frac{923520}{923521} \times\left(\prod_{i=1}^{m} \frac{q_{i}}{q_{i}-1}\right)<\frac{923520}{923521} \times \frac{1771561}{1771560} \times e^{\gamma} \times \log \log n<e^{\gamma} \times \log \log n
$$

since $\frac{923520}{923521} \times \frac{1771561}{1771560}<1$. In light of lemma 3.4 and the fact that $e^{e^{23.762143}}<10^{10^{10}}$, we then conclude that Robins $(n)$ holds for $n>5040$ when $v_{31}(n) \leq 3$.

\section{A Particular Case}

We can easily prove that Robins $(n)$ is true for certain kind of numbers:

Lemma 4.1 Robins $(n)$ holds for $n>5040$ when $q \leq 7$, where $q$ is the largest prime divisor of $n$.

Proof Let $n>5040$ and let all its prime divisors be $q_{1}<\cdots<q_{m} \leq 5$, then we need to prove

$$
f(n)<e^{\gamma} \times \log \log n
$$

that is true when

$$
\prod_{i=1}^{m} \frac{q_{i}}{q_{i}-1} \leq e^{\gamma} \times \log \log n
$$


according to the lemma 2.1. For $q_{1}<\cdots<q_{m} \leq 5$,

$$
\prod_{i=1}^{m} \frac{q_{i}}{q_{i}-1} \leq \frac{2 \times 3 \times 5}{1 \times 2 \times 4}=3.75<e^{\gamma} \times \log \log (5040) \approx 3.81 .
$$

However, we know for $n>5040$

$$
e^{\gamma} \times \log \log (5040)<e^{\gamma} \times \log \log n
$$

and therefore, the proof is complete when $q_{1}<\cdots<q_{m} \leq 5$. The remaining case is for $n>5040$ when all its prime divisors are $q_{1}<\cdots<q_{m} \leq 7$. Robins $(n)$ holds for $n>5040$ when $v_{7}(n) \leq 6$ according to the lemma 3.1 [5]. Hence, it is enough to prove this for those natural numbers $n>5040$ when $7^{7} \mid n$. For $q_{1}<\cdots<q_{m} \leq 7$,

$$
\prod_{i=1}^{m} \frac{q_{i}}{q_{i}-1} \leq \frac{2 \times 3 \times 5 \times 7}{1 \times 2 \times 4 \times 6}=4.375<e^{\gamma} \times \log \log \left(7^{7}\right) \approx 4.65 .
$$

However, for $n>5040$ and $7^{7} \mid n$, we know that

$$
e^{\gamma} \times \log \log \left(7^{7}\right) \leq e^{\gamma} \times \log \log n
$$

and as a consequence, the proof is complete when $q_{1}<\cdots<q_{m} \leq 7$.

\section{A Better Bound}

This is a known result:

Lemma 5.1 [8]. For $x>1$ :

$$
\sum_{q \leq x} \frac{1}{q}<\log \log x+B+\frac{1}{\log ^{2} x}
$$

where

$$
B=0.2614972128 \cdots
$$

denotes the (Meissel-)Mertens constant [8].

We show a better result:

Lemma 5.2 For $x \geq 11$, we have

$$
\sum_{q \leq x} \frac{1}{q}<\log \log x+\gamma-0.12
$$

Proof Let's define $H=\gamma-B$. The lemma 5.1 is the same as

$$
\sum_{q \leq x} \frac{1}{q}<\log \log x+\gamma-\left(H-\frac{1}{\log ^{2} x}\right) .
$$

For $x \geq 11$,

and thus,

$$
\left(H-\frac{1}{\log ^{2} x}\right)>\left(0.31-\frac{1}{\log ^{2} 11}\right)>0.12
$$

$$
\sum_{q \leq x} \frac{1}{q}<\log \log x+\gamma-\left(H-\frac{1}{\log ^{2} x}\right)<\log \log x+\gamma-0.12
$$


6 On a Square Free Number

We know the following results:

Lemma 6.1 [3]. For $0<a<b$ :

$$
\frac{\log b-\log a}{b-a}=\frac{1}{(b-a)} \int_{a}^{b} \frac{d t}{t}>\frac{1}{b} .
$$

Lemma 6.2 [3]. For $q>0$ :

$$
\log (q+1)-\log q=\int_{q}^{q+1} \frac{d t}{t}<\frac{1}{q} .
$$

We recall that an integer $n$ is said to be square free if for every prime divisor $q$ of $n$ we have $q^{2} \nmid n$ [3]. Robins $(n)$ holds for all $n>5040$ that are square free [3].

Lemma 6.3 For a square free number

$$
n=q_{1} \times \cdots \times q_{m}
$$

such that $q_{1}<q_{2}<\cdots<q_{m}$ are odd prime numbers, $q_{m} \geq 11$ and $3 \nmid n$, then:

$$
\frac{\pi^{2}}{6} \times \frac{3}{2} \times \sigma(n) \leq e^{\gamma} \times n \times \log \log \left(2^{19} \times n\right) .
$$

Proof By induction with respect to $\omega(n)$, that is the number of distinct prime factors of $n$ [3]. Put $\omega(n)=m$ [3]. We need to prove the assertion for those integers with $m=1$. From a square free number $n$, we obtain

$$
\sigma(n)=\left(q_{1}+1\right) \times\left(q_{2}+1\right) \times \cdots \times\left(q_{m}+1\right)
$$

when $n=q_{1} \times q_{2} \times \cdots \times q_{m}$ [3]. In this way, for every prime number $q_{i} \geq 11$, then we need to prove

$$
\frac{\pi^{2}}{6} \times \frac{3}{2} \times\left(1+\frac{1}{q_{i}}\right) \leq e^{\gamma} \times \log \log \left(2^{19} \times q_{i}\right) .
$$

For $q_{i}=11$, we have

$$
\frac{\pi^{2}}{6} \times \frac{3}{2} \times\left(1+\frac{1}{11}\right) \leq e^{\gamma} \times \log \log \left(2^{19} \times 11\right)
$$

is actually true. For another prime number $q_{i}>11$, we have

$$
\left(1+\frac{1}{q_{i}}\right)<\left(1+\frac{1}{11}\right)
$$

and

$$
\log \log \left(2^{19} \times 11\right)<\log \log \left(2^{19} \times q_{i}\right)
$$

which clearly implies that the inequality (6.4) is true for every prime number $q_{i} \geq 11$. Now, suppose it is true for $m-1$, with $m \geq 2$ and let us consider the assertion for those 
square free $n$ with $\omega(n)=m$ [3]. So let $n=q_{1} \times \cdots \times q_{m}$ be a square free number and assume that $q_{1}<\cdots<q_{m}$ for $q_{m} \geq 11$.

Case 1: $q_{m} \geq \log \left(2^{19} \times q_{1} \times \cdots \times q_{m-1} \times q_{m}\right)=\log \left(2^{19} \times n\right)$.

By the induction hypothesis we have

$\frac{\pi^{2}}{6} \times \frac{3}{2} \times\left(q_{1}+1\right) \times \cdots \times\left(q_{m-1}+1\right) \leq e^{\gamma} \times q_{1} \times \cdots \times q_{m-1} \times \log \log \left(2^{19} \times q_{1} \times \cdots \times q_{m-1}\right)$

and hence

$$
\begin{gathered}
\frac{\pi^{2}}{6} \times \frac{3}{2} \times\left(q_{1}+1\right) \times \cdots \times\left(q_{m-1}+1\right) \times\left(q_{m}+1\right) \leq \\
e^{\gamma} \times q_{1} \times \cdots \times q_{m-1} \times\left(q_{m}+1\right) \times \log \log \left(2^{19} \times q_{1} \times \cdots \times q_{m-1}\right)
\end{gathered}
$$

when we multiply the both sides of the inequality by $\left(q_{m}+1\right)$. We want to show

$$
\begin{gathered}
e^{\gamma} \times q_{1} \times \cdots \times q_{m-1} \times\left(q_{m}+1\right) \times \log \log \left(2^{19} \times q_{1} \times \cdots \times q_{m-1}\right) \leq \\
e^{\gamma} \times q_{1} \times \cdots \times q_{m-1} \times q_{m} \times \log \log \left(2^{19} \times q_{1} \times \cdots \times q_{m-1} \times q_{m}\right)=e^{\gamma} \times n \times \log \log \left(2^{19} \times n\right) .
\end{gathered}
$$

Indeed the previous inequality is equivalent with

$q_{m} \times \log \log \left(2^{19} \times q_{1} \times \cdots \times q_{m-1} \times q_{m}\right) \geq\left(q_{m}+1\right) \times \log \log \left(2^{19} \times q_{1} \times \cdots \times q_{m-1}\right)$

or alternatively

$$
\begin{gathered}
\frac{q_{m} \times\left(\log \log \left(2^{19} \times q_{1} \times \cdots \times q_{m-1} \times q_{m}\right)-\log \log \left(2^{19} \times q_{1} \times \cdots \times q_{m-1}\right)\right)}{\log q_{m}} \geq \\
\frac{\log \log \left(2^{19} \times q_{1} \times \cdots \times q_{m-1}\right)}{\log q_{m}} .
\end{gathered}
$$

We can apply the inequality in lemma 6.1 just using $b=\log \left(2^{19} \times q_{1} \times \cdots \times q_{m-1} \times\right.$ $\left.q_{m}\right)$ and $a=\log \left(2^{19} \times q_{1} \times \cdots \times q_{m-1}\right)$. Certainly, we have

$$
\begin{gathered}
\log \left(2^{19} \times q_{1} \times \cdots \times q_{m-1} \times q_{m}\right)-\log \left(2^{19} \times q_{1} \times \cdots \times q_{m-1}\right)= \\
\log \frac{2^{19} \times q_{1} \times \cdots \times q_{m-1} \times q_{m}}{2^{19} \times q_{1} \times \cdots \times q_{m-1}}=\log q_{m} .
\end{gathered}
$$

In this way, we obtain

$$
\begin{gathered}
\frac{q_{m} \times\left(\log \log \left(2^{19} \times q_{1} \times \cdots \times q_{m-1} \times q_{m}\right)-\log \log \left(2^{19} \times q_{1} \times \cdots \times q_{m-1}\right)\right)}{\log q_{m}}> \\
\frac{q_{m}}{\log \left(2^{19} \times q_{1} \times \cdots \times q_{m}\right)} .
\end{gathered}
$$

Using this result we infer that the original inequality is certainly satisfied if the next inequality is satisfied

$$
\frac{q_{m}}{\log \left(2^{19} \times q_{1} \times \cdots \times q_{m}\right)} \geq \frac{\log \log \left(2^{19} \times q_{1} \times \cdots \times q_{m-1}\right)}{\log q_{m}}
$$


which is trivially true for $q_{m} \geq \log \left(2^{19} \times q_{1} \times \cdots \times q_{m-1} \times q_{m}\right)$ [3].

Case 2: $q_{m}<\log \left(2^{19} \times q_{1} \times \cdots \times q_{m-1} \times q_{m}\right)=\log \left(2^{19} \times n\right)$.

We need to prove

$$
\frac{\pi^{2}}{6} \times \frac{3}{2} \times \frac{\sigma(n)}{n} \leq e^{\gamma} \times \log \log \left(2^{19} \times n\right) .
$$

We know $\frac{3}{2}<1.503<\frac{4}{2.66}$. Nevertheless, we could have

$$
\frac{3}{2} \times \frac{\sigma(n)}{n} \times \frac{\pi^{2}}{6}<\frac{4 \times \sigma(n)}{3 \times n} \times \frac{\pi^{2}}{2 \times 2.66}
$$

and therefore, we only need to prove

$$
\frac{\sigma(3 \times n)}{3 \times n} \times \frac{\pi^{2}}{5.32} \leq e^{\gamma} \times \log \log \left(2^{19} \times n\right)
$$

where this is possible because of $3 \nmid n$. If we apply the logarithm to the both sides of the inequality, then we obtain

$\log \left(\frac{\pi^{2}}{5.32}\right)+(\log (3+1)-\log 3)+\sum_{i=1}^{m}\left(\log \left(q_{i}+1\right)-\log q_{i}\right) \leq \gamma+\log \log \log \left(2^{19} \times n\right)$.

In addition, note that $\log \left(\frac{\pi^{2}}{5.32}\right)<\frac{1}{2}+0.12$. However, we know

$$
\gamma+\log \log q_{m}<\gamma+\log \log \log \left(2^{19} \times n\right)
$$

since $q_{m}<\log \left(2^{19} \times n\right)$. We use that lemma 6.2 for each term $\log (q+1)-\log q$ and thus,

$$
0.12+\frac{1}{2}+\frac{1}{3}+\frac{1}{q_{1}}+\cdots+\frac{1}{q_{m}} \leq 0.12+\sum_{q \leq q_{m}} \frac{1}{q} \leq \gamma+\log \log q_{m}
$$

where $q_{m} \geq 11$. Hence, it is enough to prove

$$
\sum_{q \leq q_{m}} \frac{1}{q} \leq \gamma+\log \log q_{m}-0.12
$$

but this is true according to the lemma 5.2 for $q_{m} \geq 11$. In this way, we finally show the lemma is indeed satisfied.

\section{Robin on Divisibility}

Robins $(n)$ holds for every $n>5040$ that is not divisible by 2 [3]. We extend this property to other prime numbers:

Lemma 7.1 Robins $(n)$ holds for all $n>5040$ when $3 \nmid n$. More precisely: every possible counterexample $n>5040$ of the Robin inequality must comply with $\left(2^{20} \times 3^{13}\right) \mid$ $n$. 
Proof We will check the Robin inequality is true for every natural number $n=$ $q_{1}^{a_{1}} \times q_{2}^{a_{2}} \times \cdots \times q_{m}^{a_{m}}>5040$ such that $q_{1}, q_{2}, \cdots, q_{m}$ are distinct prime numbers, $a_{1}, a_{2}, \cdots, a_{m}$ are natural numbers and $3 \nmid n$. We know this is true when the greatest prime divisor of $n>5040$ is lesser than or equal to 7 according to the lemma 4.1. Therefore, the remaining case is when the greatest prime divisor of $n>5040$ is greater than or equal to 11 . We need to prove

$$
f(n)<e^{\gamma} \times \log \log n
$$

that is true when

$$
\frac{\pi^{2}}{6} \times \prod_{i=1}^{m} \frac{q_{i}+1}{q_{i}} \leq e^{\gamma} \times \log \log n
$$

according to the lemma 2.3. Using the formula (6.3) for the square free numbers, then we obtain that is equivalent to

$$
\frac{\pi^{2}}{6} \times \frac{\sigma\left(n^{\prime}\right)}{n^{\prime}} \leq e^{\gamma} \times \log \log n
$$

where $n^{\prime}=q_{1} \times \cdots \times q_{m}$ is the square free kernel of the natural number $n$ [3]. The Robin inequality has been proved for all integers $n$ not divisible by 2 (which are bigger than 10) [3]. Hence, we only need to prove the Robin inequality is true when $2 \mid n^{\prime}$. In addition, we know that Robins $(n)$ holds for every $n>5040$ when $v_{2}(n) \leq 19$ according to the lemma 3.1 [5]. Consequently, we only need to prove that $\operatorname{Robins}(n)$ holds for $n>5040$ when $2^{20} \mid n$ and thus,

$$
e^{\gamma} \times n^{\prime} \times \log \log \left(2^{19} \times \frac{n^{\prime}}{2}\right) \leq e^{\gamma} \times n^{\prime} \times \log \log n
$$

because of $2^{19} \times \frac{n^{\prime}}{2} \leq n$ where $2^{20} \mid n$ and $2 \mid n^{\prime}$. So,

$$
\frac{\pi^{2}}{6} \times \sigma\left(n^{\prime}\right) \leq e^{\gamma} \times n^{\prime} \times \log \log \left(2^{19} \times \frac{n^{\prime}}{2}\right) .
$$

According to the formula (6.3) for the square free numbers and $2 \mid n^{\prime}$, then,

$$
\frac{\pi^{2}}{6} \times 3 \times \sigma\left(\frac{n^{\prime}}{2}\right) \leq e^{\gamma} \times 2 \times \frac{n^{\prime}}{2} \times \log \log \left(2^{19} \times \frac{n^{\prime}}{2}\right)
$$

which is the same as

$$
\frac{\pi^{2}}{6} \times \frac{3}{2} \times \sigma\left(\frac{n^{\prime}}{2}\right) \leq e^{\gamma} \times \frac{n^{\prime}}{2} \times \log \log \left(2^{19} \times \frac{n^{\prime}}{2}\right)
$$

where this is true according to the lemma 6.3 when $3 \nmid \frac{n^{\prime}}{2}$. In addition, we know that $\operatorname{Robins}(n)$ holds for every $n>5040$ when $v_{3}(n) \leq 12$ according to the lemma 3.1 [5]. Hence, we only need to prove that Robins $(n)$ holds for every $n>5040$ when $2^{20} \mid n$ and $3^{13} \mid n$. To sum up, the proof is complete.

Let's state the following known properties: 
Lemma 7.2 $\sigma(n)$ and $f(n)$ are multiplicatives [3]. Besides, for a prime number $q$ and a positive integer $a \geq 0$, we have that $\sigma\left(q^{a}\right)=\frac{q^{a+1}-1}{q-1}[3]$. We know that $f\left(q^{a}\right)<$ $\frac{q}{q-1}$ and $f\left(q^{a+1}\right)>f\left(q^{a}\right)$ for all primes $q$ and all $a \geq 0$.

Lemma 7.3 Robins $(n)$ holds for all $n>5040$ when $5 \nmid n$ or $7 \nmid n$.

Proof We need to prove

$$
f(n)<e^{\gamma} \times \log \log n
$$

when $\left(2^{20} \times 3^{13}\right) \mid n$. Suppose that $n=2^{a} \times 3^{b} \times m$, where $a \geq 20, b \geq 13,2 \nmid m, 3 \nmid m$ and $5 \nmid m$ or $7 \nmid m$. Therefore, we need to prove

$$
f\left(2^{a} \times 3^{b} \times m\right)<e^{\gamma} \times \log \log \left(2^{a} \times 3^{b} \times m\right) .
$$

We know

$$
f\left(2^{a} \times 3^{b} \times m\right)=f\left(3^{b}\right) \times f\left(2^{a} \times m\right)
$$

since $f$ is multiplicative [3]. In addition, we know $f\left(3^{b}\right)<\frac{3}{2}$ for every natural number $b$ [3]. In this way, we have

$$
f\left(3^{b}\right) \times f\left(2^{a} \times m\right)<\frac{3}{2} \times f\left(2^{a} \times m\right) .
$$

However, that would be equivalent to

$$
\frac{3}{2} \times f\left(2^{a} \times m\right)=\frac{9}{8} \times f(3) \times f\left(2^{a} \times m\right)=\frac{9}{8} \times f\left(2^{a} \times 3 \times m\right)
$$

where $f(3)=\frac{4}{3}$ since $f$ is multiplicative [3]. Nevertheless, we have

$$
\frac{9}{8} \times f\left(2^{a} \times 3 \times m\right)<f(5) \times f\left(2^{a} \times 3 \times m\right)=f\left(2^{a} \times 3 \times 5 \times m\right)
$$

and

$$
\frac{9}{8} \times f\left(2^{a} \times 3 \times m\right)<f(7) \times f\left(2^{a} \times 3 \times m\right)=f\left(2^{a} \times 3 \times 7 \times m\right)
$$

where $5 \nmid m$ or $7 \nmid m, f(5)=\frac{6}{5}$ and $f(7)=\frac{8}{7}$. We know the Robin inequality is true for $2^{a} \times 3 \times 5 \times m$ and $2^{a} \times 3 \times 7 \times m$ when $a \geq 20$, since this is true for every natural number $n>5040$ when $v_{3}(n) \leq 12$ according to the lemma 3.1 [5]. Hence, we would have

$$
f\left(2^{a} \times 3 \times 5 \times m\right)<e^{\gamma} \times \log \log \left(2^{a} \times 3 \times 5 \times m\right)<e^{\gamma} \times \log \log \left(2^{a} \times 3^{b} \times m\right)
$$

and

$$
f\left(2^{a} \times 3 \times 7 \times m\right)<e^{\gamma} \times \log \log \left(2^{a} \times 3 \times 7 \times m\right)<e^{\gamma} \times \log \log \left(2^{a} \times 3^{b} \times m\right)
$$

when $b \geq 13$.

Lemma 7.4 Robins $(n)$ holds for all $n>5040$ when a prime number $11 \leq q \leq 47$ complies with $q \nmid n$. 
Proof We know that Robins $(n)$ holds for every $n>5040$ when $v_{7}(n) \leq 6$ according to the lemma 3.1 [5]. We need to prove

$$
f(n)<e^{\gamma} \times \log \log n
$$

when $\left(2^{20} \times 3^{13} \times 7^{7}\right) \mid n$. Suppose that $n=2^{a} \times 3^{b} \times 7^{c} \times m$, where $a \geq 20, b \geq 13$, $c \geq 7,2 \nmid m, 3 \nmid m, 7 \nmid m, q \nmid m$ and $11 \leq q \leq 47$. Therefore, we need to prove

$$
f\left(2^{a} \times 3^{b} \times 7^{c} \times m\right)<e^{\gamma} \times \log \log \left(2^{a} \times 3^{b} \times 7^{c} \times m\right) .
$$

We know

$$
f\left(2^{a} \times 3^{b} \times 7^{c} \times m\right)=f\left(7^{c}\right) \times f\left(2^{a} \times 3^{b} \times m\right)
$$

since $f$ is multiplicative [3]. In addition, we know $f\left(7^{c}\right)<\frac{7}{6}$ for every natural number $c$ [3]. In this way, we have

$$
f\left(7^{c}\right) \times f\left(2^{a} \times 3^{b} \times m\right)<\frac{7}{6} \times f\left(2^{a} \times 3^{b} \times m\right) .
$$

However, that would be equivalent to

$$
\frac{7}{6} \times f\left(2^{a} \times 3^{b} \times m\right)=\frac{49}{48} \times f(7) \times f\left(2^{a} \times 3^{b} \times m\right)=\frac{49}{48} \times f\left(2^{a} \times 3^{b} \times 7 \times m\right)
$$

where $f(7)=\frac{8}{7}$ since $f$ is multiplicative [3]. In addition, we know

$$
\frac{49}{48} \times f\left(2^{a} \times 3^{b} \times 7 \times m\right)<f(q) \times f\left(2^{a} \times 3^{b} \times 7 \times m\right)=f\left(2^{a} \times 3^{b} \times 7 \times q \times m\right)
$$

where $q \nmid m, f(q)=\frac{q+1}{q}$ and $11 \leq q \leq 47$. Nevertheless, we know the Robin inequality is true for $2^{a} \times 3^{b} \times 7 \times q \times m$ when $a \geq 20$ and $b \geq 13$, since this is true for every natural number $n>5040$ when $v_{7}(n) \leq 6$ according to the lemma 3.1 [5]. Hence, we would have

$$
\begin{aligned}
f\left(2^{a} \times 3^{b} \times 7 \times q \times m\right) & <e^{\gamma} \times \log \log \left(2^{a} \times 3^{b} \times 7 \times q \times m\right) \\
& <e^{\gamma} \times \log \log \left(2^{a} \times 3^{b} \times 7^{c} \times m\right)
\end{aligned}
$$

when $c \geq 7$ and $11 \leq q \leq 47$.

Lemma 7.5 Robins $(n)$ holds for all $n>5040$ when a prime number $53 \leq q \leq 953$ complies with $q \nmid n$.

Proof We know that Robins $(n)$ holds for every $n>5040$ when $v_{31}(n) \leq 3$ according to the lemma 3.5. We need to prove that

$$
f(n)<e^{\gamma} \times \log \log n
$$

when $\left(2^{20} \times 3^{13} \times 31^{4}\right) \mid n$. Suppose that $n=2^{a} \times 3^{b} \times 31^{c} \times m$, where $a \geq 20, b \geq 13$, $c \geq 4,2 \nmid m, 3 \nmid m, 31 \nmid m, q \nmid m$ and $53 \leq q \leq 953$. Therefore, we need to prove that

$$
f\left(2^{a} \times 3^{b} \times 31^{c} \times m\right)<e^{\gamma} \times \log \log \left(2^{a} \times 3^{b} \times 31^{c} \times m\right) .
$$


We know that

$$
f\left(2^{a} \times 3^{b} \times 31^{c} \times m\right)=f\left(31^{c}\right) \times f\left(2^{a} \times 3^{b} \times m\right)
$$

since $f$ is multiplicative [3]. In addition, we know that $f\left(31^{c}\right)<\frac{31}{30}$ for every natural number $c$ [3]. In this way, we have that

$$
f\left(31^{c}\right) \times f\left(2^{a} \times 3^{b} \times m\right)<\frac{31}{30} \times f\left(2^{a} \times 3^{b} \times m\right) .
$$

However, that would be equivalent to

$\frac{31}{30} \times f\left(2^{a} \times 3^{b} \times m\right)=\frac{961}{960} \times f(31) \times f\left(2^{a} \times 3^{b} \times m\right)=\frac{961}{960} \times f\left(2^{a} \times 3^{b} \times 31 \times m\right)$

where $f(31)=\frac{32}{31}$ since $f$ is multiplicative [3]. In addition, we know that

$\frac{961}{960} \times f\left(2^{a} \times 3^{b} \times 31 \times m\right)<f(q) \times f\left(2^{a} \times 3^{b} \times 31 \times m\right)=f\left(2^{a} \times 3^{b} \times 31 \times q \times m\right)$

where $q \nmid m, f(q)=\frac{q+1}{q}$ and $53 \leq q \leq 953$. Nevertheless, we know the Robin inequality is true for $2^{a} \times 3^{b} \times 31 \times q \times m$ when $a \geq 20$ and $b \geq 13$, since this is true for every natural number $n>5040$ when $v_{31}(n) \leq 3$ according to the lemma 3.5. Hence, we would have that

$$
\begin{aligned}
f\left(2^{a} \times 3^{b} \times 31 \times q \times m\right) & <e^{\gamma} \times \log \log \left(2^{a} \times 3^{b} \times 31 \times q \times m\right) \\
& <e^{\gamma} \times \log \log \left(2^{a} \times 3^{b} \times 31^{c} \times m\right)
\end{aligned}
$$

when $c \geq 4$ and $53 \leq q \leq 953$

\section{Helpful Lemmas}

In mathematics, the Chebyshev function $\theta(x)$ is given by

$$
\theta(x)=\sum_{q \leq x} \log q
$$

where $q \leq x$ means all the prime numbers $q$ that are less than or equal to $x$.

Lemma 8.1 [8]. For $x \geq 41$ :

$$
\theta(x)>\left(1-\frac{1}{\log (x)}\right) \times x .
$$

Besides, we know that

Lemma 8.2 [8]. For $x \geq 286$ :

$$
\prod_{q \leq x} \frac{q}{q-1}<e^{\gamma} \times\left(\log x+\frac{1}{2 \times \log (x)}\right) .
$$

For the counting prime function $\pi(x)$, we know that 
Lemma 8.3 [8]. For $x \geq 17$ :

$$
\frac{x}{\log x}<\pi(x)<1.25506 \times \frac{x}{\log x} .
$$

The following lemma is crucial in our proof

Lemma 8.4 [6]. For $x>-1$ :

$$
\frac{x}{x+1} \leq \log (1+x) \leq x .
$$

The smallest counterexample of the Robin inequality greater than 5040 complies with

Lemma 8.5 If $n>5040$ is the smallest counterexample of the Robin inequality, then $q<\log n$ where $q$ denotes the largest prime factor of $n$ [3].

We show some tools that could help us in the final proof.

Lemma 8.6 Let $q \geq 2$ be a prime and let $b \geq 0$ be a positive integer. If $q^{a} \| n$, then

$$
f\left(q^{b} \times n\right)=f(n) \times \frac{q^{a+b+1}-1}{q^{a+b+1}-q^{b}}
$$

where $q^{a} \| n$ signifies that $q^{a}$ divides $n$, but $q^{a+1}$ does not divide $n$.

Proof We assume that $q^{a} \| n$. Since $\sigma(n)$ and $f(n)$ are multiplicatives according to the lemma 7.2, then we would only need to study $f\left(q^{a+b}\right)$ where we know from lemma 7.2 that $\sigma\left(q^{a}\right)=\frac{q^{a+1}-1}{q-1}$. Then,

$$
\begin{aligned}
f\left(q^{a+b}\right) & =\frac{q^{a+b+1}-1}{q^{a+b} \times(q-1)} \times \frac{q^{a+1}-1}{q^{a} \times(q-1)} \times \frac{q^{a} \times(q-1)}{q^{a+1}-1} \\
& =f\left(q^{a}\right) \times \frac{q^{a+b+1}-1}{q^{a+b} \times(q-1)} \times \frac{q^{a} \times(q-1)}{q^{a+1}-1} \\
& =f\left(q^{a}\right) \times \frac{q^{a+b+1}-1}{q^{b}} \times \frac{1}{q^{a+1}-1} \\
& =f\left(q^{a}\right) \times \frac{q^{a+b+1}-1}{q^{a+b+1}-q^{b}} .
\end{aligned}
$$

Let's see another inequalities:

Lemma 8.7 If $n>5040$ is the smallest counterexample of the Robin inequality, then

$$
\frac{\log \log n}{\log q}<\left(1+\frac{1}{2 \times \log ^{2} q}\right)
$$

and

$$
\frac{\log \log \log n}{\log q}<\frac{\log \log q}{\log q}+\frac{1}{2 \times \log ^{3} q}
$$

when we assume that $q \geq 953$ is the largest prime factor of $n$. 
Proof Let $\prod_{i=1}^{m} q_{i}^{a_{i}}$ be the representation of $n$ as a product of the first $m$ consecutive primes $q_{1}<\cdots<q_{m}$ with natural numbers as exponents $a_{1}, \ldots, a_{m}$. According to the theorems 1.3 and 1.4, the primes $q_{1}<\cdots<q_{m}$ must be the first $m$ consecutive primes since $n>5040$ should be an Hardy-Ramanujan integer. We assume that $q_{m} \geq 953$. For $q_{m} \geq 953$, we have that

$$
\prod_{q \leq q_{m}} \frac{q}{q-1}<e^{\gamma} \times\left(\log q_{m}+\frac{1}{2 \times \log \left(q_{m}\right)}\right)
$$

because of the lemma 8.2. We use that lemma 2.1 to show that

$$
e^{\gamma} \times \log \log n \leq f(n)<\prod_{q \leq q_{m}} \frac{q}{q-1}<e^{\gamma} \times\left(\log q_{m}+\frac{1}{2 \times \log \left(q_{m}\right)}\right)
$$

since we assume that $n$ is a counterexample of the Robin inequality. In this way, we obtain that

$$
\log \log n<\left(\log q_{m}+\frac{1}{2 \times \log \left(q_{m}\right)}\right)
$$

which is the same as

$$
\frac{\log \log n}{\log q_{m}}<\left(1+\frac{1}{2 \times \log ^{2}\left(q_{m}\right)}\right) .
$$

Besides, if we apply the logarithm to the both sides of the inequality, then

$$
\log \log \log n<\log \left(\log q_{m} \times\left(1+\frac{1}{2 \times \log ^{2}\left(q_{m}\right)}\right)\right)
$$

that is equivalent to

$$
\log \log \log n<\log \log q_{m}+\log \left(1+\frac{1}{2 \times \log ^{2}\left(q_{m}\right)}\right) .
$$

We use that lemma 8.4 to show that

$$
\log \left(1+\frac{1}{2 \times \log ^{2}\left(q_{m}\right)}\right) \leq \frac{1}{2 \times \log ^{2}\left(q_{m}\right)} .
$$

Therefore, we finally have that

$$
\frac{\log \log \log n}{\log q_{m}}<\frac{\log \log q_{m}}{\log q_{m}}+\frac{1}{2 \times \log ^{3} q_{m}} .
$$

Let's show another inequality

Lemma 8.8 For $q_{m} \geq 953$, we have that

$$
\sum_{q \leq q_{m}} \frac{\log \log q}{q_{m}}>\frac{1}{\log q_{m}}
$$


Proof This is the same as

$$
\sum_{q \leq q_{m}} \log \log q>\frac{q_{m}}{\log q_{m}} .
$$

According to the lemma 8.3, it is enough to show that

$$
\sum_{q \leq q_{m}} \log \log q \geq \pi\left(q_{m}\right)>\frac{q_{m}}{\log q_{m}}
$$

when $q_{m} \geq 953$. We know that for all primes $q_{i}>q_{m} \geq 953$, then

$$
\log \log q_{i}>1 \text {. }
$$

Hence, it is enough to prove that

$$
\sum_{q \leq q_{m}} \log \log q \geq \sum_{q \leq 953} \log \log q \geq \pi(953) .
$$

We compute that

$$
\sum_{q \leq 953} \log \log q>274 .
$$

However, we know that $q_{274}=1759>953$ and thus,

$$
274 \geq \pi(953) \text {. }
$$

Therefore, the proof is done.

\section{Proof of Main Theorems}

Theorem 9.1 Robins $(n)$ holds for all $n>5040$ when a prime number $q \leq 953$ complies with $q \nmid n$.

Proof This is a compendium of the results from the theorem 1.2 and the lemmas 7.1, 7.3, 7.4 and 7.5.

Theorem 9.2 Let $\prod_{i=1}^{m} q_{i}^{a_{i}}$ be the representation of $n$ as a product of the first $m$ consecutive primes $q_{1}<\cdots<q_{m}$ with natural numbers as exponents $a_{1}, \ldots, a_{m}$. We obtain a contradiction just assuming that $n>5040$ is the smallest integer such that Robins $(n)$ does not hold.

Proof According to the theorems 1.3 and 1.4, the primes $q_{1}<\cdots<q_{m}$ must be the first $m$ consecutive primes since $n>5040$ should be an Hardy-Ramanujan integer. From the theorem 9.1, we know that necessarily $q_{m} \geq 953$. Under our assumption, we know that

$$
f(n) \geq e^{\gamma} \times \log \log n .
$$

For $b=1$ and the lemma 8.6, we know that

$$
f(n)=f\left(q_{i} \times m\right)=f(m) \times \frac{q_{i}^{a_{i}+2}-1}{q_{i}^{a_{i}+2}-q_{i}}
$$


for every prime $q_{i}$ that divides $n$ where $m=\frac{n}{q_{i}}$. If we subtract $f(m)$ to both sides of the inequality, then we obtain that

$$
f(n)-f(m) \geq e^{\gamma} \times \log \log n-f(m) .
$$

Then,

$$
\begin{aligned}
f(n)-f(m) & =f(m) \times \frac{q_{i}^{a_{i}+2}-1}{q_{i}^{a_{i}+2}-q_{i}}-f(m) \\
& =f(m) \times\left(\frac{q_{i}^{a_{i}+2}-1}{q_{i}^{a_{i}+2}-q_{i}}-1\right) \\
& =f(m) \times\left(\frac{q_{i}-1}{q_{i}^{a_{i}+2}-q_{i}}\right) \\
& =f(m) \times\left(\frac{q_{i}-1}{q_{i} \times\left(q_{i}^{a_{i}+1}-1\right)}\right) \\
& =f(m) \times\left(\frac{1}{q_{i} \times \sigma\left(q_{i}^{a_{i}}\right)}\right) \\
& =f\left(m^{\prime}\right) \times f\left(q_{i}^{a_{i}-1}\right) \times\left(\frac{1}{q_{i} \times \sigma\left(q_{i}^{a_{i}}\right)}\right) \\
& =f\left(m^{\prime}\right) \times \frac{\sigma\left(q_{i}^{a_{i}-1}\right)}{q_{i}^{a_{i}-1}} \times\left(\frac{1}{q_{i} \times \sigma\left(q_{i}^{a_{i}}\right)}\right) \\
& <f\left(m^{\prime}\right) \times \frac{\sigma\left(q_{i}^{a_{i}}\right)}{q_{i}^{a_{i}}} \times\left(\frac{1}{q_{i} \times \sigma\left(q_{i}^{a_{i}}\right)}\right) \\
& =f\left(m^{\prime}\right) \times \frac{1}{q_{i}^{a_{i}+1}}
\end{aligned}
$$

where $m^{\prime}=\frac{n}{q_{i}^{a_{i}}}$ and we know that $q_{i}^{a_{i}} \| n$ and $\frac{\sigma\left(q_{i}^{a_{i}}\right)}{q_{i}^{a_{i}}}>\frac{\sigma\left(q_{i}^{a_{i}-1}\right)}{q_{i}^{a_{i}-1}}$ because of the lemma 7.2. In this way, we have that

$$
f\left(m^{\prime}\right) \times \frac{1}{q_{i}^{a_{i}+1}} \geq e^{\gamma} \times \log \log n-f(m) .
$$

We know that Robins $\left(m^{\prime}\right)$ and Robins $(m)$ hold, since $n>5040$ is the smallest integer such that Robins $(n)$ does not hold. Consequently, we only need to prove that

$$
\begin{aligned}
e^{\gamma} \times \log \log m^{\prime} \times \frac{1}{q_{i}^{a_{i}+1}} & >f\left(m^{\prime}\right) \times \frac{1}{q_{i}^{a_{i}+1}} \\
& \geq e^{\gamma} \times \log \log n-f(m) \\
& >e^{\gamma} \times \log \log n-e^{\gamma} \times \log \log m .
\end{aligned}
$$

As result, we have that

$$
\log \log m^{\prime} \times \frac{1}{q_{i}^{a_{i}+1}}>\log \log \left(q_{i} \times m\right)-\log \log m
$$


since $m=\frac{n}{q_{i}}$. We know that

$$
\begin{aligned}
\log \log \left(q_{i} \times m\right)-\log \log m & =\log \left(\log q_{i}+\log m\right)-\log \log m \\
& =\log \left(\log m \times\left(1+\frac{\log q_{i}}{\log m}\right)\right)-\log \log m \\
& =\log \log m+\log \left(1+\frac{\log q_{i}}{\log m}\right)-\log \log m \\
& =\log \left(1+\frac{\log q_{i}}{\log m}\right) .
\end{aligned}
$$

In addition, we know that

$$
\log \left(1+\frac{\log q_{i}}{\log m}\right) \geq \frac{\log q_{i}}{\log n}
$$

using the lemma 8.4. Certainly, we will have that

$$
\log \left(1+\frac{\log q_{i}}{\log m}\right) \geq \frac{\frac{\log q_{i}}{\log m}}{\log q_{i}}+1=\frac{\log q_{i}}{\log m}=\frac{\log q_{i}}{\log q_{i}+\log m} .
$$

As a consequence, we would have

$$
\log \log m^{\prime} \times \frac{1}{q_{i}^{a_{i}+1}}>\frac{\log q_{i}}{\log n}
$$

which is equivalent to

$$
\log n \times \log \log m^{\prime}>q_{i}^{a_{i}+1} \times \log q_{i} .
$$

However, we know that

$$
\log n \times \log \log n>\log n \times \log \log m^{\prime}
$$

and thus

$$
\log n \times \log \log n>q_{i}^{a_{i}+1} \times \log q_{i} .
$$

For $n>10^{10^{10}}$, we have that $\log n \times \log \log n>1$ according to the lemma 3.4. Moreover, for $q_{i} \geq 3$, then $q_{i}^{a_{i}+1} \times \log q_{i}>1$. In addition, for $q_{1}=2$, we have that $q_{1}^{a_{1}+1} \times$ $\log q_{1}>1$ since $a_{1} \geq 20$ due to the lemma 3.1. Since the both sides of the inequality is greater that 1 for all primes $q_{i}$ which divides $n$, then we can multiply the inequalities to obtain

$$
(\log n \times \log \log n)^{\pi\left(q_{m}\right)}>n \times N_{m} \times \prod_{i=1}^{m} \log q_{i}
$$

where $N_{m}=\prod_{i=1}^{m} q_{i}$ is the primorial number of order $m$. If we apply the logarithm to the both sides of the inequality, then we would have

$$
\pi\left(q_{m}\right) \times(\log \log n+\log \log \log n)>\log n+\log N_{m}+\sum_{i=1}^{m} \log \log q_{i}
$$


which is equivalent to

$$
\pi\left(q_{m}\right) \times(\log \log n+\log \log \log n)>\log n+\theta\left(q_{m}\right)+\sum_{i=1}^{m} \log \log q_{i} .
$$

If we apply the lemma 8.3 , then we would have

$$
1.25506 \times \frac{q_{m}}{\log q_{m}} \times(\log \log n+\log \log \log n)>\log n+\theta\left(q_{m}\right)+\sum_{i=1}^{m} \log \log q_{i} .
$$

Let's introduce the lemma 8.1 in this inequality and thus

$$
1.25506 \times \frac{q_{m}}{\log q_{m}} \times(\log \log n+\log \log \log n)>\log n+\left(1-\frac{1}{\log q_{m}}\right) \times q_{m}+\sum_{i=1}^{m} \log \log q_{i} .
$$

In addition, we can transform this into

$$
1.25506 \times \frac{q_{m}}{\log q_{m}} \times(\log \log n+\log \log \log n)>q_{m}+\left(1-\frac{1}{\log q_{m}}\right) \times q_{m}+\sum_{i=1}^{m} \log \log q_{i}
$$

because of the lemma 8.5 . If we divide the both sides by $q_{m}$, then

$$
1.25506 \times \frac{1}{\log q_{m}} \times(\log \log n+\log \log \log n)>1+1-\frac{1}{\log q_{m}}+\sum_{i=1}^{m} \frac{\log \log q_{i}}{q_{m}} .
$$

According to the lemma 8.8, we know that

$$
-\frac{1}{\log q_{m}}+\sum_{i=1}^{m} \frac{\log \log q_{i}}{q_{m}}=\alpha>0 .
$$

Consequently, we would have that

$$
1.25506 \times\left(\frac{\log \log n}{\log q_{m}}+\frac{\log \log \log n}{\log q_{m}}\right)>2+\alpha .
$$

If we use the lemma 8.7 , then

$$
1.25506 \times\left(1+\frac{1}{2 \times \log ^{2} q_{m}}+\frac{\log \log q_{m}}{\log q_{m}}+\frac{1}{2 \times \log ^{3} q_{m}}\right)>2+\alpha .
$$

We know that

$$
\begin{aligned}
& 1.25506 \times\left(1+\frac{1}{2 \times \log ^{2} q_{m}}+\frac{\log \log q_{m}}{\log q_{m}}+\frac{1}{2 \times \log ^{3} q_{m}}\right) \\
& \leq 1.25506 \times\left(1+\frac{1}{2 \times \log ^{2} 953}+\frac{\log \log 953}{\log 953}+\frac{1}{2 \times \log ^{3} 953}\right)
\end{aligned}
$$

and we have that

$$
1.25506 \times\left(1+\frac{1}{2 \times \log ^{2} 953}+\frac{\log \log 953}{\log 953}+\frac{1}{2 \times \log ^{3} 953}\right) \approx 1.62266460495 .
$$


Consequently, we have that

$$
2>1.25506 \times\left(1+\frac{1}{2 \times \log ^{2} q_{m}}+\frac{\log \log q_{m}}{\log q_{m}}+\frac{1}{2 \times \log ^{3} q_{m}}\right)>2+\alpha>2
$$

and

$$
2>2
$$

is a contradiction. To sum up, we obtain a contradiction just assuming that $n>5040$ is the smallest integer such that $\operatorname{Robins}(n)$ does not hold.

Theorem 9.3 Robins $(n)$ holds for all $n>5040$.

Proof Due to the theorem 9.2, we can assure there is not any natural number $n>5040$ such that Robins $(n)$ does not hold.

Theorem 9.4 The Riemann Hypothesis is true.

Proof This is a direct consequence of theorems 1.1 and 9.3

\section{Acknowledgments}

I thank Richard J. Lipton for helpful comments.

\section{References}

1. Akbary, A., Friggstad, Z.: Superabundant numbers and the Riemann hypothesis. The American Mathematical Monthly 116(3), 273-275 (2009). DOI doi:10.4169/193009709X470128

2. Alaoglu, L., Erdős, P.: On highly composite and similar numbers. Transactions of the American Mathematical Society 56(3), 448-469 (1944). DOI doi:10.2307/1990319

3. Choie, Y., Lichiardopol, N., Moree, P., Solé, P.: On Robin's criterion for the Riemann hypothesis. Journal de Théorie des Nombres de Bordeaux 19(2), 357-372 (2007). DOI doi:10.5802/jtnb.591

4. Edwards, H.M.: Riemann's Zeta Function. Dover Publications (2001)

5. Hertlein, A.: Robin's Inequality for New Families of Integers. Integers 18 (2018)

6. Kozma, L.: Useful Inequalities. http://www.lkozma.net/inequalities_cheat_sheet/ineq. pdf (2021). Accessed on 2021-09-27

7. Robin, G.: Grandes valeurs de la fonction somme des diviseurs et hypothèse de Riemann. J. Math. pures appl 63(2), 187-213 (1984)

8. Rosser, J.B., Schoenfeld, L.: Approximate Formulas for Some Functions of Prime Numbers. Illinois Journal of Mathematics 6(1), 64-94 (1962). DOI doi:10.1215/ijm/1255631807 